\title{
Aortic root abscess - a deadly complication of UTI-induced infective endocarditis
}

\author{
Rohan Anand MBA, Jasmin Rahesh MBA, Carlos Morales MD, Pooja Sethi MD
}

\begin{abstract}
Aortic root abscess and endocarditis should be suspected in patients with bradycardia and sepsis. We present a case of a 76-year-old man who presented with a urinary tract infection and sepsis and developed bradycardia and ventricular standstill during his hospital admission. A transthoracic echocardiogram was unrevealing; transesophageal echocardiogram showed prosthetic valve dehiscence, an aortic root abscess, an intracardiac fistula, and tricuspid valve endocarditis. This case highlights the importance of suspecting endocarditis in patients with sepsis and a known source of infection, especially if blood cultures do not clear or conduction abnormalities develop.
\end{abstract}

Keywords: aortic root abscess, periannular abscess, heart block, infective endocarditis

\section{INTRODUCTION}

Aortic root abscess arising from periannular valvular abscess is one of the most feared and lethal sequelae of infective endocarditis (IE) and cardiac valvular surgery. ${ }^{1}$ Complications include complete heart block, aortocavitary fistula, and destruction of the affected valve..$^{2,3}$ Its insidious presentation can delay its diagnosis unless the clinician has a high index of suspicion; its rapid extension into and destruction of critical cardiac components confer significant morbidity and mortality, presenting a highly complex challenge for surgical repair.

\section{CASE}

A 76-year-old man with an extensive past medical history, including coronary artery disease status post coronary artery bypass grafting, aortic stenosis

Corresponding author: Rohan Anand Contact Information: Rohan.anand@ttuhsc.edu DOI: $10.12746 /$ swrccc.v9i41.933 status post replacement with a bioprosthetic valve, and chronic atrial fibrillation (AF), was admitted by the emergency department for a urinary tract infection (UTI) and was evaluated for AF with persistent bradycardia. The patient developed lethargy, fevers, and delirium with blood cultures positive for Enterococcus faecalis. Transthoracic echocardiogram (TTE) on postadmission day (PAD) 11 did not show any signs of endocarditis. Despite treatment with IV daptomycin and penicillin G, blood cultures remained positive, prompting suspicion of endocarditis possibly complicated by a valvular abscess. Due to the patient's persistent bradycardia (35-45 bpm) interspersed with ventricular standstill and complete heart block (Figure 1), a transvenous pacemaker was placed on PAD 19, stabilizing the patient's heart rate at $70 \mathrm{bpm}$. Subsequent transesophageal echocardiogram (TEE) demonstrated endocarditis of the prosthetic aortic valve with root abscess, an intracardiac fistula, and associated tricuspid endocarditis with severe tricuspid regurgitation (Figure 2-3). The consulted cardiothoracic surgeon recommended transfer of care to a higher level of care for aortic valve and root replacement and repair of the intracardiac fistula, and the patient was transferred from our facility on PAD 21. The patient has been 


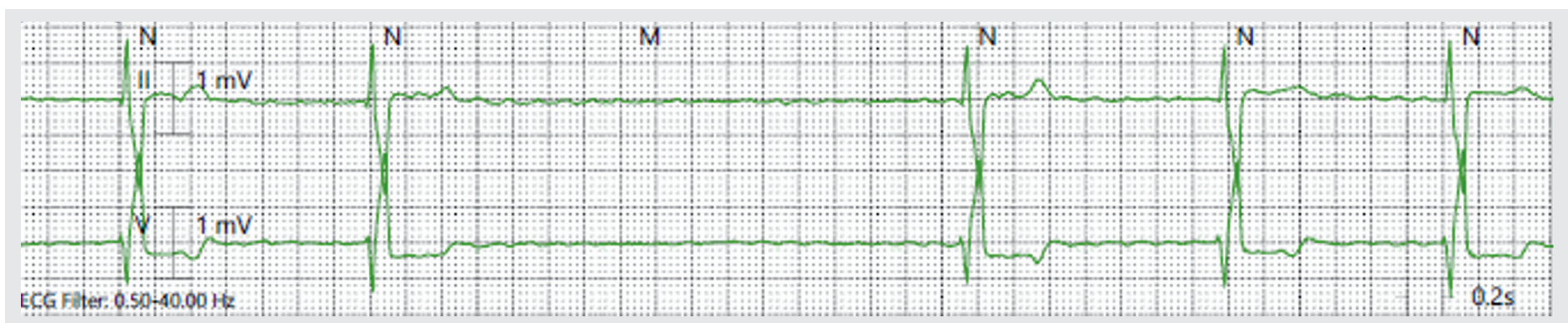

Figure 1. Patient ECG demonstrating atrial fibrillation with ventricular standstill.

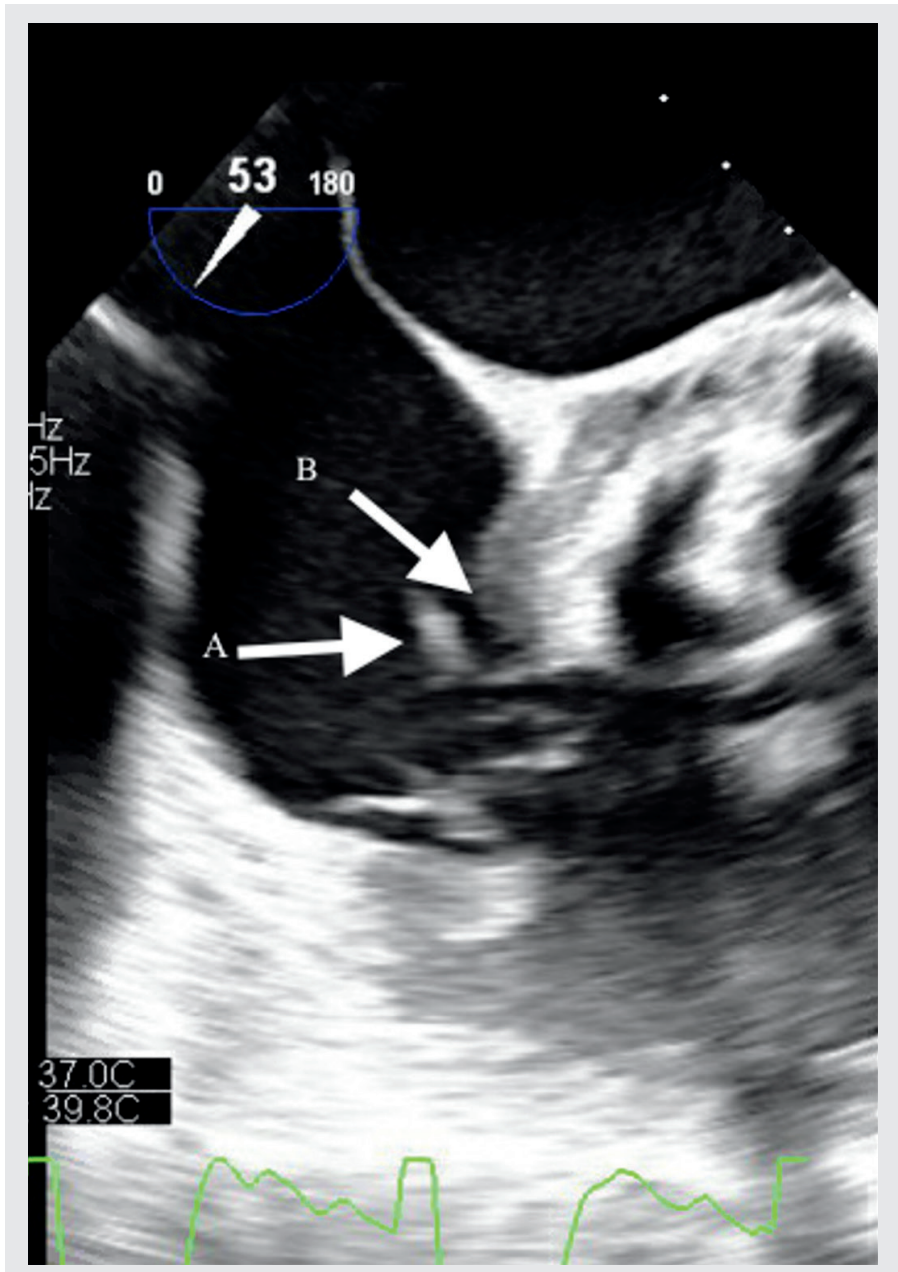

Figure 2. A $0.4 \times 1.1 \mathrm{~cm}$ flailing lesion attached to the tricuspid valve ( $\operatorname{arrowhead} \mathrm{A})$, with a $1.2 \times 1.3 \mathrm{~cm}$ lesion on the septal leaflet of the tricuspid valve (arrowhead B). informed and has provided consent for his case to be published in this report.

\section{Discussion}

Infective endocarditis, already a disease of high morbidity and mortality, may be complicated by a range of sequelae that further worsen patient outcomes. Periannular abscess is a particularly dangerous complication of IE, occurring in up to $46 \%$ of IE cases involving the aortic valve. ${ }^{1}$ The aortic valve is most commonly complicated by primary abscess formation, with the mitral and tricuspid valves being significantly less commonly involved. ${ }^{4}$ Abscess formation is more likely in prosthetic valve endocarditis than in native valve endocarditis, probably due to the

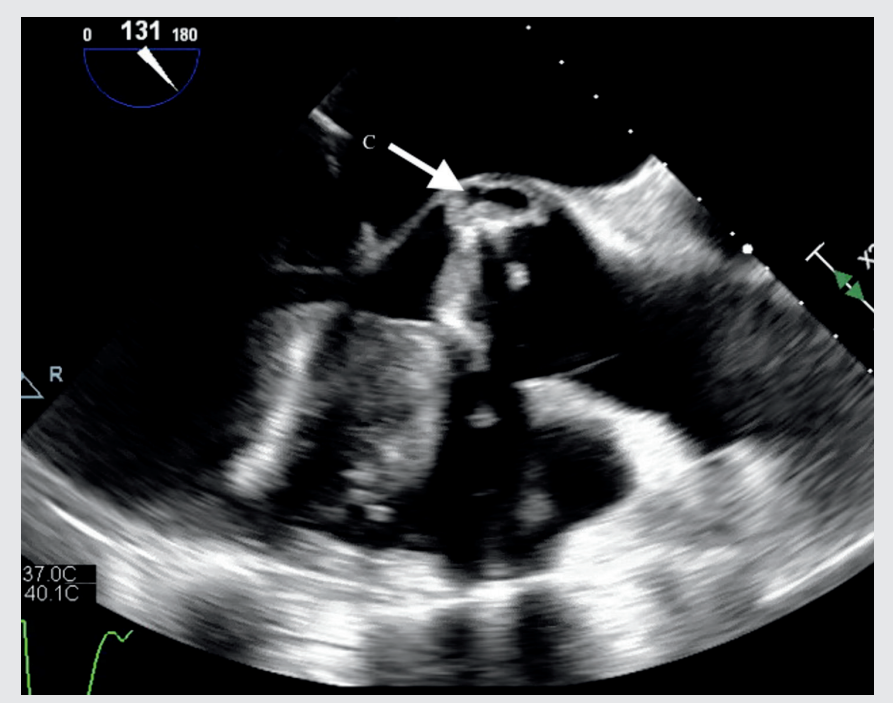

Figure 3. Aortic root abscess. 
higher risk of valve dehiscence, with the former carrying a higher mortality. Staphylococcus aureus and $S$. epidermidis are the most implicated organisms, followed by Streptococcus spp. and Enterococcus spp. ${ }^{2}$ Destruction of tissue beyond the valve annulus may result in aortic root abscess, aortocavitary fistulae, and heart failure..$^{2,3}$

The clinical manifestations that suggest a case of IE has developed a periannular abscess are subtle. Due to the aortic valve's close proximity to the intraventricular septum containing the proximal ventricular conduction system, conduction abnormalities-most commonly $3^{\text {rd }}$ degree AV block-may be the first sign of abscess formation resulting in the erosion of critical conductive tissue. Bacteremia that does not respond to appropriate antibiotic therapy also suggests a resistant infection.

Imaging should be obtained in all patients suspected of abscess formation to ascertain the extent of infection and the affected structures to guide surgical repair. While TTE has excellent specificity (99\%) to screen for abscess formation, its sensitivity is quite poor. ${ }^{5}$ Transesophageal echocardiography TEE is the most sensitive modality $(87 \%)$ by which the aortic valve and its surrounding structure may be visualized in high detail. Evidence of abscess formation includes localized reduced echodensity, echolucent cavities within or adjacent to cardiac structures, and perivalvular erratic rocking motion.

Surgery is often the only means by which full eradication of the abscess can be achieved; complete resolution by antibiotics alone is quite rare. All cases require radical debridement of all infected tissue and reconstruction of destroyed structures before proceeding to valve replacement. Aortic allografts, homografts, mechanical and biologic prosthetic valves are suitable options. ${ }^{6-8}$ The ideal choice for valve prosthesis is controversial. Leyh et al. found that the survival rates of patients with aortic valve endocarditis repaired with aortic homograft were not dependent on whether a homograft or composite prosthesis was used. ${ }^{9}$ Similarly, Moon et al. found that outcomes including operative mortality, survival rate, and recurrence of IE were independent of the choice of a mechanical or bioprosthetic valve. ${ }^{10}$ The choice of valve replacement may ultimately depend on the extent of damage, the surgeon's expertise and comfort with the repair, and the preference of the center treating the patient.

Our case is notable for the patient's presenting with a relatively common source of infection (here a UTI) that quickly developed into bacteremia and sepsis. The subsequent development of bradycardia with heart block and ventricular standstill, particularly in a patient with known bioprosthetic valve, should prompt clinicians to have a high index of suspicion for IE with valvular abscess development. As with our patient, TEE should be performed without delay to guide emergent surgical debridement and repair.

\section{ConcLusion}

Bacteremia and sepsis are relatively common in the hospital ward and can arise from a wide variety of infectious sites. A history of valve replacement places such patients at risk for IE; the addition of conduction abnormalities on ECG suggests extension of IE to form an abscess at the valvular apparatus. Prompt evaluation with TEE is essential to plan for surgical repair.

Article citation: Anand R, Rahesh J, Morales C, Sethi P. Aortic root abscess - a deadly complication of UTI-induced infective endocarditis. The Southwest Respiratory and Critical Care Chronicles 2021;9(41):50-53 From: Department of Surgery (RA, JR) and Department of Internal Medicine (CM, PS), Texas Tech University Health Sciences Center, Lubbock, Texas

Submitted: $8 / 22 / 2021$

Accepted: 9/22/2021

Reviewer: Aliakbar Arvandi MD

Conflicts of interest: none

This work is licensed under a Creative Commons Attribution-Share A like 4.0 International License.

\section{REFERENCES}

1. Mahmoud K, Hammouda T, Kandil H, et al. Prevalence and predictors of aortic root abscess among patients with left-sided infective endocarditis: a cross-sectional comparative study. 
The Egyptian Heart Journal 2020;72(1):62. DOI: 10.1186/ s43044-020-00098-6

2. Anguera I, Miro JM, Vilacosta I, et al. Aorto-cavitary fistulous tract formation in infective endocarditis: clinical and echocardiographic features of 76 cases and risk factors for mortality. Eur Heart J 2005;26(3):288-97.

3. Okada K, Tanaka H, Takahashi H, et al. Aortic root replacement for destructive aortic valve endocarditis with left ventricular-aortic discontinuity. Ann Thorac Surg 2008;85(3): 940-5.

4. Arnett EN, Roberts WC. Valve ring abscess in active infective endocarditis. Frequency, location, and clues to clinical diagnosis from the study of 95 necropsy patients. Circulation 1976;54(1):140-5.

5. Daniel WG, Mügge $A$, Martin RP, et al. Improvement in the diagnosis of abscesses associated with endocarditis by transesophageal echocardiography. N Engl J Med 1991;324(12): 795-800.
6. Leontyev S, Borger MA, Modi P, et al. Surgical management of aortic root abscess: a 13-year experience in 172 patients with 100\% follow-up. J Thorac Cardiovasc Surg 2012;143(2): 332-7.

7. Musci M, Hübler M, Amiri A, et al. Surgical treatment for active infective prosthetic valve endocarditis: 22-year singlecentre experience. Eur J Cardiothorac Surg. 2010;38(5): 528-38.

8. Kirali K, Sarikaya S, Ozen Y, et al. Surgery for aortic root abscess: a 15-year experience. Tex Heart Inst J 2016;43(1):20-8.

9. Leyh RG, Knobloch K, Hagl C, et al. Replacement of the aortic root for acute prosthetic valve endocarditis: prosthetic composite versus aortic allograft root replacement. J Thorac Cardiovasc Surg 2004;127(5):1416-20.

10. Moon MR, Miller DC, Moore KA, et al. Treatment of endocarditis with valve replacement: the question of tissue versus mechanical prosthesis. Ann Thorac Surg 2001;71(4): 1164-71. 